\title{
Metabolism of ciclesonide in the upper and lower airways: review of available data
}

\author{
Ruediger Nave \\ Nigel McCracken
}

Nycomed GmbH, Konstanz, Germany
Correspondence: Ruediger Nave Department Pharmacometrics/ Pharmacokinetics, Nycomed GmbH, Byk-Gulden Straße 2, 78467 Konstanz, Germany

$\mathrm{Tel}+49753 \mid 842362$

Fax +4975318492362

Email ruediger.nave@nycomed.com

\begin{abstract}
Ciclesonide is a novel corticosteroid (CS) for the treatment of asthma and allergic rhinitis. After administration, the parent compound ciclesonide is converted by intracellular airway esterases to its pharmacologically active metabolite desisobutyryl-ciclesonide (des-CIC). We investigated the in vitro activation of ciclesonide and further esterification of des-CIC to (mainly) des-CIC oleate in several human target organ test systems. Human precision-cut lung slices, alveolar type II epithelial cells (A549), normal bronchial epithelial cells (NHBE), and nasal epithelial cells (HNEC) were incubated with ciclesonide. Enzymes characterization and the determination of the reversibility of fatty acid esterification was investigated in HNEC and NHBE. Ciclesonide was taken up and converted to des-CIC in all cellular test systems. Intracellular concentrations of des-CIC were maintained for up to $24 \mathrm{~h}$. Formation of des-CIC oleate increased over time in HNEC, A549 cells, and lung slices. The formed des-CIC fatty acid conjugates were reconverted to des-CIC. Increasing concentrations of carboxylesterase and cholinesterase inhibitors progressively reduced the formation of metabolites. The results derived from these studies demonstrate the activation of ciclesonide to des-CIC in the upper and lower airways. The reversible formation of des-CIC fatty acid conjugates may prolong the anti-inflammatory activity of des-CIC and may allow for once-daily dosing.
\end{abstract}

Keywords: ciclesonide, des-CIC, metabolism, human, lung, nasal tissue

\section{Introduction}

Asthma is one of the most common chronic inflammatory disorders of the airways and is characterized by airway obstruction, inflammation and hyper-responsiveness resulting from complex interactions among inflammatory cells, mediators, and the cells and tissues of the airways. International and national treatment guidelines recommend the use of inhaled corticosteroids (CS) as first-line therapy for patients with asthma (NIH 1997; GINA 2005).

Inhaled CS effectively treat asthma due to their potent anti-inflammatory effect, altering the production of mediators involved in airway inflammation and hyperresponsiveness (GINA 2005; Barnes 1990). The reductions in airway inflammation and hyper-responsiveness improve lung function and asthma symptoms, and reduce acute exacerbations requiring urgent care and rescue medication use (Blais et al 1998; Georgitis 1999; GINA 2005). In addition, early intervention with inhaled CS in persistent asthma has been shown to improve clinical outcomes, which is thought to be due to their beneficial effects on airway remodeling (NIH 1997; Canonica 2006).

Allergic rhinitis is defined as intermittent (or seasonal) or persistent (or perennial) and affects $10 \%$ to $30 \%$ of the world's population (Skoner 2001; Berger 2003; CKS 2007). The most common symptoms of allergic rhinitis include sneezing, itching, rhinorrhea, and nasal blockage. According to the Allergic Rhinitis and its Impact on Asthma guidelines, allergic rhinitis is a major chronic respiratory disease that is increasing in prevalence; reduces quality of life, performance, and productivity; and is a significant 
risk factor for asthma (Bousquet et al 2001, 2004). Intranasal $\mathrm{CS}$ are the most effective treatment option in patients with moderate or severe allergic rhinitis and provide effective relief of nasal symptoms and congestion because of their potent anti-inflammatory activity (Gelfand 2004; Storms 2004).

Ciclesonide is a new-generation CS currently developed and approved for the treatment of asthma and allergic rhinitis. Ciclesonide is administered via a metered-dose inhaler using hydrofluoroalkane (HFA) as the propellant or in a hypotonic formulation as nasal spray. In contrast to other CS that bind directly to the corticosteroid receptor, eg, budesonide, ciclesonide is a prodrug with almost no receptor binding affinity. Intracellular airway esterases convert ciclesonide to its pharmacologically active metabolite desisobutyryl-ciclesonide (des-CIC), which has a 100-fold higher binding affinity for the corticosteroid receptor than the prodrug (Dietzel et al 2001; Dent 2002).

Another feature that is associated with an improved efficacy of CS is the ability to form highly lipophilic fatty acid esters. The conjugation of a corticosteroid with fatty acids in the pulmonary tissue provides a mechanism by which the drug can be retained in the lung for a prolonged period of time (Edsbäcker and Brattsand 2002). A hydroxyl group at position $\mathrm{C}-21$ is required for the formation of the ester bond between the corticosteroid and the fatty acid (Tunek et al 1997). Budesonide and des-CIC have a hydroxyl group at the $\mathrm{C}-21$ position and conjugates with oleic and palmitic acid have been found in vitro in rat trachea and lung tissues (Nave et al 2004; Lexmüller et al 2007). Uptake and metabolism of ciclesonide are illustrated in Figure 1.

The aim is to review the metabolism data supporting the activation of ciclesonide and esterification of des-CIC and to discuss the clinical implications this may have on asthma and allergic rhinitis treatment.

\section{Methods}

All methods described in this section are briefly summarized as they are previously published.

Human nasal epithelial cells (HNEC) in culture dishes were incubated for $1 \mathrm{~h}$ at $37{ }^{\circ} \mathrm{C}$ with growth medium containing ciclesonide $\left(1 \times 10^{-7} \mathrm{~mol} / \mathrm{L}\right)$. Cells were

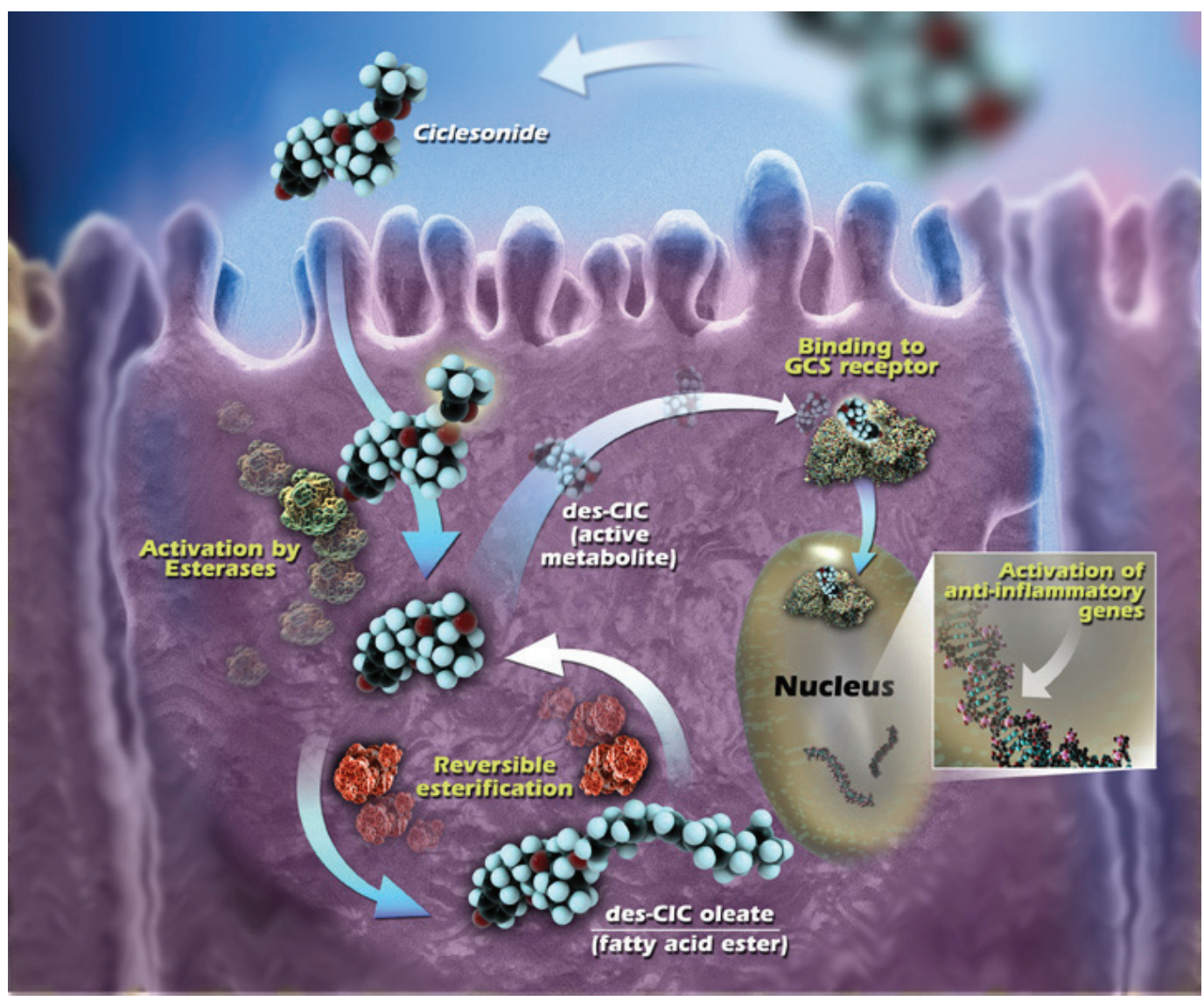

Figure I Intracellular activation of ciclesonide and reversible esterification of des-CIC. Reprinted from Nave R, MeyerW, Fuhst R, et al 2005b. Formation of fatty acid conjugates of ciclesonide active metabolite in the rat lung after 4-week inhalation of ciclesonide. Pulm Pharmacol Ther, 18:390-6. Copyright (C) Elsevier.

Abbreviations: GCS, glucocorticosteroid; des-CIC, desisobutyryl-ciclesonide. 
washed and incubated for a further $0.5,3,6$, and 24 in ciclesonide-free medium (Sato et al 2007a). To investigate reversibility of des-CIC, HNEC were incubated with $10^{-6}$ $\mathrm{mol} / \mathrm{L}$ des-CIC for $6 \mathrm{~h}$. Cells were washed and incubated in des-CIC-free medium for 3, 6, and $24 \mathrm{~h}$. Cells were collected at $0,3,6$, and $24 \mathrm{~h}$; medium was collected at $1,3,6$, and 24 h (Sato et al 2007a).

Normal human bronchial epithelial (NHBE) cells were cultured in culture dishes and incubated for up to $24 \mathrm{~h}$ with ciclesonide $\left(5 \times 10^{-6} \mathrm{~mol} / \mathrm{L}\right)$ (Mutch et al 2007). To investigate reversibility of des-CIC, NHBE were incubated with des-CIC $\left(1 \times 10^{-6} \mathrm{~mol} / \mathrm{L}\right)$ for $6 \mathrm{~h}$. Cells were washed and incubated in des-CIC free medium up to $24 \mathrm{~h}$. Cells were collected at $0,3,6$ and $24 \mathrm{~h}$; medium was collected at $1,3,6$ and $24 \mathrm{~h}$ and replaced by fresh medium at 1,3 and $6 \mathrm{~h}$ (Nonaka unpublished data).

Human alveolar type II epithelial (A549) cells in culture dishes were incubated for $3,5,10,20$, or 30 min (uptake), or for $1 \mathrm{~h}$ (metabolism) at $37^{\circ} \mathrm{C}$ with growth medium containing ciclesonide $\left(2 \times 10^{-8} \mathrm{~mol} / \mathrm{L}\right)$. To investigate metabolism, cells were washed and incubated for a further 3,6 , and 24 in ciclesonide-free medium (Nonaka et al 2007).

Human precision-cut lung slices were incubated at $37{ }^{\circ} \mathrm{C}$ with ciclesonide $\left(1 \times 10^{-5} \mathrm{~mol} / \mathrm{L}\right)$ or ${ }^{14} \mathrm{C}$-ciclesonide $\left(2.5 \times 10^{-5} \mathrm{~mol} / \mathrm{L}\right)$ (Nave et al 2006a, 2007).

To identify the esterases involved HNEC and NHBE were preincubated for $30 \mathrm{~min}$ with the chemical inhibitors (final concentration $1 \times 10^{-5} \mathrm{~mol} / \mathrm{L}$ for the inhibition of esterases) paraoxon, bis(p-nitrophenyl)phosphate (BNPP), tetraisopropyl pyrophosphoramide (iso-OMPA), eserine, 4-hydroxymercuribenzoic acid sodium salt (PMB), and ethylenediamine tetraacetic acid (EDTA).

Ciclesonide $\left(5 \times 10^{-6} \mathrm{~mol} / \mathrm{L}\right)$ was added to start the reaction and cells were incubated for 2 (NHBE) or $6 \mathrm{~h}$ (HNEC) (Mutch et al 2007; Sato et al 2007a).

For all investigations, ethanolic extracts of harvested cells or tissues were analyzed using high performance liquid chromatography (UPLC) with ultraviolet (UV), radio, or tandem mass spectrometric (LC-MS/MS) detection. In general for all cell test systems, ethanol was added to each medium harvested, and this mixture was vortex-mixed, centrifuged, and the extracted supernatant was harvested. After cells frozen in the plates were gradually thawed, the cells were frozen by liquid nitrogen again. After the freeze/thaw cycle was repeated again, ethanol containing $0.1 \%$ Tween 80 was added to each well in order to extract the analyte from cells. The samples were centrifuged and the extracted supernatant was harvested. For lung slices, samples of the incubation medium were prepared by mixing the same volume of medium with a solution $\left(0.2 \mathrm{M} \mathrm{ZnSO}_{4}\right.$ /acetonitrile, $\left.1: 1\right)$ for precipitation. Following centrifugation, the supernatant was assayed by HPLC. Tissue samples were homogenized in methanol using ultrasonic treatment and were centrifuged. After centrifugation, supernatant was dried and resuspended in methanol before analysis.

Concentrations of ciclesonide, des-CIC, and des-CIC fatty acid conjugates (des-CIC-oleate and des-CIC-palmitate) in the sample extracted from the culture medium, cells, or slices were analyzed by liquid chromatography with a UV detector monitoring at $242 \mathrm{~nm}$ or with LC-MS/MS. Ethanol solutions containing ciclesonide, des-CIC, des-CIC-oleate, and des-CIC-palmitate were used as standard solutions. Deuterated des-CIC was used as an internal standard for LCMS/MS. Water/ethanol gradient systems were run and these systems were suitable for assaying the parent compounds and the metabolites.

\section{Results}

\section{Cellular uptake of ciclesonide}

A fast uptake of ciclesonide was confirmed in all cellular test systems. The earliest timepoint used was $3 \mathrm{~min}$ in the investigation with A549 cells. The uptake of ciclesonide in A549 cells was almost complete within 10 min after the start of the incubation period (Table 1) (Nonaka et al 2007).

The uptake of ciclesonide into human lung and liver slices was investigated using radiolabeled ciclesonide. Quantification of radiolabeled ciclesonide and its metabolites within the tissue were possible following combustion (Nave et al 2006a). At late timepoints levels of radioactive drug-related material in human lung tissue were 7-fold higher than in the liver tissue. In the lung slices, recovered radioactivity was up to $60 \%$ of the spiked amount of drug.

Table I Uptake of ciclesonide into A549 cells (derived from data of Nonaka et al 2007)

\begin{tabular}{lll}
\hline $\begin{array}{l}\text { Incubation time } \\
(\mathbf{m i n})\end{array}$ & $\begin{array}{l}\text { Mean concentration } \\
\text { (pmol/dish) }\end{array}$ & $\begin{array}{l}\text { Standard } \\
\text { deviation }\end{array}$ \\
\hline 3 & 15.51 & 2.62 \\
5 & 18.19 & 2.34 \\
10 & 37.32 & 23.51 \\
20 & 35.31 & 9.09 \\
30 & 50.33 & 5.12 \\
\hline
\end{tabular}

Notes: Intracellular concentrations of total ciclesonide (sum of ciclesonide, desisobutyryl-ciclesonide [des- $\mathrm{ClC}$ ], and des- $\mathrm{ClC}$ fatty acid esters) in A549 cells during incubation with $2 \times 10^{-8} \mathrm{M}$ ciclesonide (5 dishes per time point). 


\section{Enzymolgy of ciclesonide activation}

In the absence of exogenous inhibitors, ciclesonide was hydrolyzed to des-CIC in cellular test systems and lung tissue.

In HNEC and NHBE cells, varying concentrations of carboxylesterase- and cholinesterase-specific inhibitors (BNPP, iso-OMP) caused a dose-dependent decrease in ciclesonide metabolism (Mutch et al 2007; Sato et al 2007a). BNPP, iso-OMP, and non-specific inhibitor paraoxon $\left(1 \times 10^{-5} \mathrm{~mol} / \mathrm{L}\right)$ decreased the hydrolysis of ciclesonide to des-CIC by more than 75\% (Table 2). Arylesterase- and A-esterase-specific inhibitors (PMB, EDTA) did not affect ciclesonide metabolism in HNEC. A similar result was observed in NHBE cells (Table 2).

\section{Formation of des-CIC fatty acid esters}

In A549 cells, HNEC, and lung tissue, des-CIC was detectable up to $24 \mathrm{~h}$ post treatment.

Intracellular formation of des-CIC fatty acid esters in airway epithelial cells and lung tissue increased over time ( $24 \mathrm{~h}$ data provided in Table 3 ). A higher amount of des-CIC oleate than des-CIC palmitate was detected in all cellular systems, indicating that des-CIC oleate was the main fatty acid ester formed (Table 3).

The quantitative analysis of all metabolites formed in lung slices following incubation with ${ }^{14} \mathrm{C}$-ciclesonide confirmed the activation and formation of fatty acid esters of des-CIC (Nave et al 2006a).

\section{Reversibility of esterification}

The total amount of intracellular des-CIC fatty acid esters decreased over $24 \mathrm{~h}$ to $20 \%$ of the original concentration,

Table 2 Inhibition of esterases in HNEC and HNBE cells (derived from data of Mutch et al 2007; Sato et al 2007a)

\begin{tabular}{llll}
\hline Inhibitor & Target esterase & \multicolumn{2}{l}{$\begin{array}{l}\text { Mean } \pm \text { SEM } \\
\% \text { control activity }\end{array}$} \\
\cline { 3 - 4 } & & HNEC & NHBE \\
\hline Paraoxon & CarbE, BChE,AChE & $25.0 \pm 0.3$ & $16.7 \pm 0.7$ \\
BNPP & CarbE & $24.9 \pm 0.3$ & $20.0 \pm 3.8$ \\
Iso-OMPA & CarbE & $29.3 \pm 0.6$ & $22.3 \pm 3.8$ \\
Eserine & AChE & $48.2 \pm 0.3$ & $28.7 \pm 3.7$ \\
PMB & ArE & $103.8 \pm 0.8$ & $90.3 \pm 17.3$ \\
EDTA & A-E & $105.8 \pm 0.3$ & $78.7 \pm 4.7$ \\
\hline
\end{tabular}

Notes: data are expressed as percentage inhibition of control (no inhibitior) after incubation of cells with ciclesonide $\left(5 \times 10^{-6} \mathrm{~mol} / \mathrm{L}\right)$ and esterase inhibitors $\left(\mathrm{I} \times 10^{-6} \mathrm{~mol} / \mathrm{L}\right)$ (mean of duplicate estimations).

Abbreviations: HNEC, human bronchial epithelial cells; NHBE, normal human bronchial epithelial; CarbE, carboxylesterase; BChE, cholinesterase; $\mathrm{AChE}$, acetylcholinesterase; ArE, arylesterase; A-E, A-esterase; BNPP, bis (p-nitrophenyl) phosphate; iso-OMPA, tetraisopropyl pyrophosphoramide; PMB, p-hydroxymercuribenzoate; EDTA, ethylenediaminetetraacetic acid. whereas the total amount of des-CIC increased over time in the medium (Figure 2). The recovery at all timepoints was approximately $100 \%$, indicating that des-CIC is reconverted from des-CIC-oleate.

Similar results were obtained in HNEC (Sato et al 2007a). The total amount of des-CIC increased during the post-treatment period and accounted for approximately $85 \%$ of total analytes $24 \mathrm{~h}$ after treatment. However, the total amount of des-CIC fatty acid conjugates declined over time and represented only $15 \%$ of total analytes $24 \mathrm{~h}$ post treatment. Intracellular amounts of des-CIC and des-CIC fatty acid conjugates decreased while des-CIC progressively accumulated in the medium. Extracellular concentrations of des-CIC-oleate and des-CIC-palmitate were almost undetectable at all timepoints post-treatment.

\section{Conclusions}

The results presented here confirm that ciclesonide was effectively taken up and converted to its pharmacologically active metabolite des-CIC in vitro in human cellular test systems and human lung tissue. Despite the variety of test systems and analytical methods used, the results confirm that activation of ciclesonide occurs by a similar mechanism in the upper and lower airways. The rapid conversion of ciclesonide to des-CIC is mediated mainly by carboxylesterases and cholinesterases. The presence of des-CIC for up to $24 \mathrm{~h}$ and the reversible formation of des-CIC fatty acid esters, which serve as a slow-release pool of active drug, may prolong the anti-inflammatory activity of ciclesonide and may support a 24-h effect with once-daily dosing in patients with asthma and allergic rhinitis.

\section{Discussion}

After uptake into the cell ciclesonide, is converted to des-CIC via cleavage of an ester bond, and therefore it was postulated that esterases may be involved in the reaction. Investigations were performed to characterize the enzymology of ciclesonide metabolism in human liver microsomes. BNPP and SKF 525-A, respectively, inhibited des-CIC formation from ${ }^{14} \mathrm{C}$-ciclesonide by $82 \%$ and $49 \%$ and $\mathrm{M} 2 / \mathrm{M} 3$ (hydroxy-metabolite) formation by $82 \%-84 \%$ and $87 \%-89 \%$. It is concluded that the human hepatic metabolism of ciclesonide is primarily catalyzed by one or more esterases to desCIC and then subsequently metabolized by CYP3A4 (Peet et al 2005). Further esterase characterization was done in HNEC and NHBE cells. In both test systems carboxylesterase and cholinesterase could be identified as major esterases involved in the first step of the ciclesonide metabolism 
Table 3 Formation of des-CIC fatty acid esters in airway epithelial cells and lung tissue

\begin{tabular}{|c|c|c|c|c|c|c|c|}
\hline \multirow[t]{2}{*}{ Test system } & \multirow{2}{*}{$\begin{array}{l}\text { Substrate } \\
\text { concentration } \\
(\mathrm{mol} / \mathrm{L})\end{array}$} & \multirow{2}{*}{$\begin{array}{l}\text { Substrate } \\
\text { incubation } \\
\text { time (h) }\end{array}$} & \multirow{2}{*}{$\begin{array}{l}\text { Sample } \\
\text { collection } \\
\text { time (h) }\end{array}$} & \multicolumn{4}{|c|}{ Mean (SD) intracellular concentration ${ }^{a}$} \\
\hline & & & & $\mathrm{CIC}$ & Des-CIC & $\begin{array}{l}\text { Des-CIC- } \\
\text { oleate }\end{array}$ & $\begin{array}{l}\text { Des-CIC- } \\
\text { palmitate }\end{array}$ \\
\hline HNEC & $1 \times 10^{-7}$ & $\mathrm{Ih}$ & $24 \mathrm{~h}^{\mathrm{b}}$ & $0.31(0.07)$ & $5.39(0.99)$ & $48.43(7.46)$ & $\mathrm{I} .87(0.34)$ \\
\hline NHBE cells & $1 \times 10^{-6}$ & $\mathrm{I} \mathrm{h}$ & $24 \mathrm{~h}^{\mathrm{b}}$ & $5.22(2.53)$ & $20.05(1.55)$ & $14.10(1.38)$ & $0.59(0.19)$ \\
\hline A549 cells & $2 \times 10^{-8}$ & $\mathrm{I} \mathrm{h}$ & $24 \mathrm{~h}^{\mathrm{b}}$ & $2.3 \mathrm{I}(0.55)$ & $3.19(0.38)$ & $23.20(6.03)$ & $0.74(0.15)$ \\
\hline Lung slices & $1 \times 10^{-5}$ & $24 \mathrm{~h}$ & $24 \mathrm{~h}$ & $2.93(0.46)$ & $3.23(0.42)$ & 3.4 & \\
\hline
\end{tabular}

${ }^{a}$ Amount of ciclesonide and metabolite in HNEC (pmol/dish), NHBE (pmol/well), A549 (pmol/dish), lung tissue (nmol). Incubation in substrate-free medium.

Abbreviations: HNEC, human bronchial epithelial cells; NHBE, normal human bronchial epithelial; des-CIC, desisobutyryl-ciclesonide.

(Derived from data of Nave et al 2007; Nonaka et al 2007; Sato et al 2007a; Nonaka unpublished data).

(Mutch et al 2007; Sato et al 2007a). Esterases are known to be present in most organs of all species; however, levels can vary greatly (McCracken et al 1993).

Ciclesonide was metabolized to des-CIC in nasal mucosal homogenates of rats, guinea pigs, rabbits, and dogs without any marked differences among animal species (Sato et al 2006). In this context it should be noted that the metabolism of ciclesonide in the liver is also similar among species (Gu et al 2007).
As previously mentioned, esterases, which rapidly hydrolyze ciclesonide, are present in human lung, nose, and liver, whereas ciclesonide seemed to be more stable in human blood, skin (data not shown), and the oropharynx. After inhalation of ciclesonide via a metered dose inhaler, the bio-activation of ciclesonide to des-CIC within the oropharynx was low (Nave et al 2005a; Richter et al 2005). These findings may explain the low frequency of oropharyngeal side effects

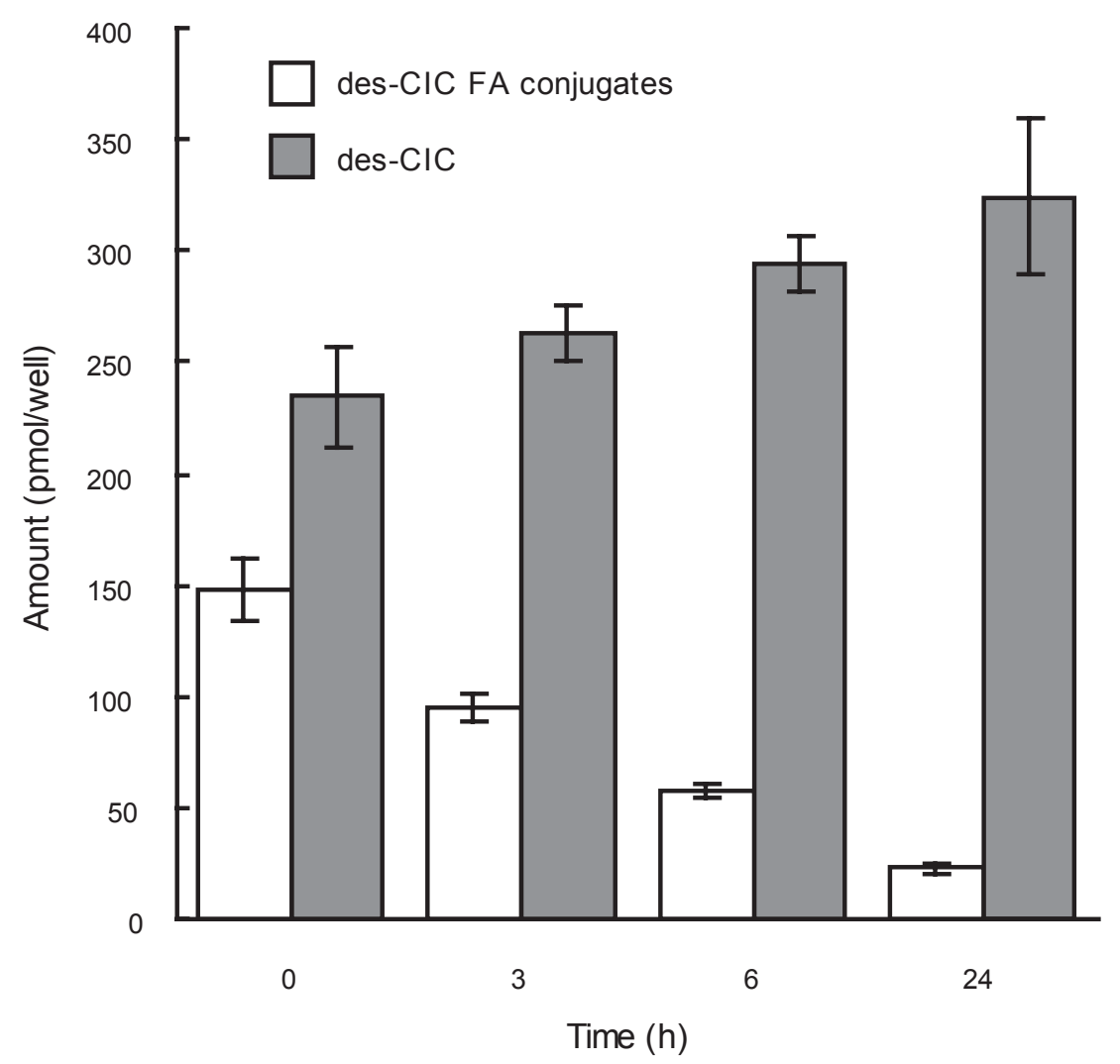

Figure 2 Reversible conjugation of des-CIC with fatty acid in human bronchial epithelial cells. Data were represented as the mean \pm SD from 6 wells. Time point of $0 \mathrm{~h}$ indicates the end of incubation with des-CIC for $6 \mathrm{~h}$. Results at $0 \mathrm{~h}$ represent only the amount in the cells and results at 3,6 , and $24 \mathrm{~h}$ represent the sum of analyte in the cells and medium harvested (Nonaka unpublished data).

Abbreviations: des-CIC, desisobutyryl-ciclesonide; des-CIC FA conjugates, sum of des-CIC-oleate and des-CIC-palmitate. 
for ciclesonide compared with other inhaled CS (Engelstaetter et al 2004; Berger 2006).

The lipophilicity and lipid conjugation are two distinct pharmacokinetic parameters that can affect the absorption rate of a CS across pulmonary and nasal membranes and influence retention time in the lung and nose. Results from various studies have confirmed that both ciclesonide and budesonide form fatty acid conjugates in a reversible manner (Edsbäcker and Brattsand 2002; Nave et al 2006a). The intracellular fatty acid conjugation of budesonide and des-CIC dramatically increased the lipophilicity. DesCIC-oleate $(\log \mathrm{D} 13.0)$ was about 5-fold more lipophilic than budesonide-oleate $(\log \mathrm{D} 12.3$ ) (Nave et al 2004). Ciclesonide and des-CIC, however, are more lipophilic than budesonide, suggesting that ciclesonide may achieve greater penetration within the target tissue.

The intracellular localization and sustained concentration of intranasal CS depend to varying levels on nasal mucosal absorption, drug lipophilicity, glucocorticoid receptor affinity, and the ability to form lipid conjugates in nasal epithelial cells (Esmailpour et al 2000). Retention and formation of fatty acid conjugates of CS in human nasal tissue and its importance in treatment of allergic rhinitis have been reported (Bonsmann et al 2001; Petersen et al 2001).

Lexmuller et al (2007) have investigated the airway retention of various glucocorticoids using an in vitro rat trachea model. It was confirmed that ciclesonide was activated to des-CIC and that fatty acid esters were formed. At $20 \mathrm{~min}$, the uptake of budesonide was similar to that of ciclesonide; however, significantly more budesonide-ester were detected compared with des-CIC-esters while the amount of active drug (budesonide and des-CIC) was comparable. This demonstrates the fast activation of ciclesonide and the subsequent fatty acid ester formation. Furthermore, rat tracheas were preincubated with the esterification inhibitor cyclandelate. Cyclandelate decreased the initial budesonide and desCIC-ester pools $(\mathrm{p}<0.01)$, and reduced the overall retention of budesonide at $3 \mathrm{~h}(\mathrm{p}<0.01)$ but not of ciclesonide. Thus, esterification as well as high lipophilicity prolongs ciclesonide airway retention.

Binding of drugs to plasma proteins is another factor that may affect the uptake of drugs into cells. Ciclesonide and des-CIC have been shown to be almost fully bound to plasma proteins (eg, albumin and $\alpha 1$-acid glycoprotein) during in vitro experiments (Rohatagi et al 2005) while budesonide has been reported to have a protein-binding rate that leaves approximately $12 \%$ of drug portion unbound (Ryrfeldt et al 1982). It was demonstrated in vitro that the cellular uptake of corticosteroids could be affected by the protein content in the cell media (Janciauskiene et al 2007). The uptake of desCIC into human hepatocytes decreased more markedly with increasing serum concentrations compared with budesonide. However, at all corticosteroid concentrations and serum levels tested, the uptake of des-CIC was significant greater than those of budesonide $(p<0.05)$. The protein content of the incubation media in most of the experiments investigating the metabolism of ciclesonide was $0.1 \%$ bovine serum albumin. Other investigations on the activation of ciclesonide in nasal tissue from various species were performed at a fixed protein concentration of $1 \mathrm{mg} / \mathrm{mL}$ (Sato et al 2006).

The in vivo situation in the nasal cavity and in the lower airways may be different to the in vitro situation using hepatocytes, as the exact protein content is not known. Furthermore, one should consider different formulations. The absorption in the lung of drugs delivered via dry powder is different compared with MDI. The formulation used for nasal administration has also been shown to influence the uptake into nasal mucosa as demonstrated for ciclesonide (Sato et al 2007b). The nasal tissue uptake and metabolism of ciclesonide were evaluated when administered as a single $143 \mu \mathrm{g}$ dose to normal Japanese white rabbits in a hypotonic versus an isotonic ciclesonide suspension. The hypotonic suspension achieved in nasal mucosa extracts higher concentrations of des-CIC (5.6-fold, 11.4-fold, and 13.4-fold) and ciclesonide (25.3-fold, 34.2-fold, and 16-fold) at 30,120 , and $240 \mathrm{~min}$ post administration. Additionally, when administered via a hypotonic suspension, des-CIC was retained up to $24 \mathrm{~h}$ post administration and fatty acid esters of des-CIC were detected in nasal mucosa. These data suggest that a hypotonic ciclesonide suspension provides higher intracellular concentrations of des-CIC up to $24 \mathrm{~h}$. Metabolism of ciclesonide in vivo in rabbit nasal mucosa confirmed the formation of des-CIC-oleate and des-CIC-palmitate conjugates of des-CIC in nasal mucosa, reaching their highest levels $8 \mathrm{~h}$ and $16 \mathrm{~h}$ post administration, respectively (Sato et al 2007b). The presence of fatty acid conjugates of des-CIC suggests that the active drug may be retained in the nasal mucosa as a reversible pool to exert sustained drug effects over time and allow once-daily dosing.

Fatty acid conjugation has also been reported for budesonide in nasal tissue (Tunek et al 1997; Petersen et al 2001). We investigated the in vitro metabolism of beclomethasone dipropionate, budesonide, ciclesonide, and fluticasone propionate in human lung precision-cut tissue slices (Nave et al 2007). In summary, the in vitro metabolic pathways of the tested CS in human lung tissue were different. While 
fluticasone propionate was metabolically stable, the majority of beclomethasone dipropionate was converted to inactive polar metabolites. The formation of fatty acid conjugates was confirmed for budesonide and des-CIC. The lipophilicity and lipid conjugation of the parent compounds and their metabolites are likely the major factors influencing their retention time in the lung (Nave et al 2007).

These data confirm the activation of ciclesonide as well as the formation of fatty acid esters in vivo in the nasal mucosa in one animal species. Furthermore, we tested the in vivo metabolism of ciclesonide in the lung by exposing rats to inhaled ciclesonide $(0.16 \mathrm{mg} / \mathrm{kg} /$ day $)$ daily for $1 \mathrm{~h}$ for a period of 4 weeks (Nave et al 2005b). Lungs were extracted with ethanol 2, 5, and $\sim 27 \mathrm{~h}$ after the final treatment. The concentrations of des-CIC and ciclesonide were determined. At the 2- and 5-h timepoints, fatty acid conjugates of des-CIC were the major metabolites (61\% and 55\%, respectively). Ciclesonide, des-CIC, and fatty acid conjugates of des-CIC were clearly present in lung samples the day after the last inhalation. This in vivo study confirmed ciclesonide activation to des-CIC and formation of fatty acid conjugates.

In vivo data for humans on the metabolism of inhaled CS in the lung are limited (Van den Brink et al 2005; Drollmann et al 2006). A recent study investigating the disposition of budesonide using samples from resected pulmonary tissue have confirmed that fatty acid esters of budesonide were formed in the human lung. Patients who were scheduled for whole lung or lobe resection for lung cancer inhaled $1000 \mu \mathrm{g}$ budesonide. Budesonide was detectable in central and peripheral lung tissue for about $10 \mathrm{~h}$ after inhalation whereas budesonide oleate was present for up to $43 \mathrm{~h}$ (Van den Brink et al 2005). After inhalation of a single dose of $1280 \mu \mathrm{g}$ ciclesonide by patients undergoing elective lung surgery, ciclesonide was rapidly hydrolyzed to des-CIC in both central lung and peripheral lung tissue. Des-CIC and des-CIC oleate were the main metabolites in both lung areas, and at least one of the metabolites of ciclesonide (des-CIC, des-CIC oleate, or des-CIC palmitate) was present in the lung tissue samples at all study time points up to $24 \mathrm{~h}$ (Drollmann et al 2006). These in vivo findings confirm that ciclesonide is activated in the human lung and that budesonide as well as des-CIC form oleate conjugates.

In summary, ciclesonide and des-CIC fatty acid esters are highly lipophilic precursors of des-CIC. Although the majority of data presented here are based on in vitro studies, there is evidence that these precursors may serve as a pool of active drug. This slow-release pool of active drug may contribute to the proven efficacy of once-daily inhaled ciclesonide in asthma therapy, as was shown in several clinical studies (Postma et al 2001; Chapman et al 2004; Langdon et al 2005). The same holds true for once-daily intranasal ciclesonide in rhinitis therapy (Nave et al 2006b; Ratner et al 2006; Kim et al 2007).

\section{Disclosures}

The authors have no conflicts of interest to declare.

\section{References}

Barnes PJ. 1990. Effect of corticosteroids on airway hyperresponsiveness. Am Rev Respir Dis, 141:S70-6.

Berger WE. 2003. Overview of allergic rhinitis. Ann Allergy Asthma Immunol, 90(Suppl):7-12.

Berger WE. 2006. Ciclesonide: A closer look at its systemic and oropharyngeal safety profile. Curr Drug Saf, 1:265-70.

Blais L, Suissa S, Boivin JF, et al. 1998. First treatment with inhaled corticosteroids and the prevention of admissions to hospital for asthma. Thorax, 53:1025-9.

Bonsmann U, Bachert C, Delank KW, et al. 2001. Presence of fluticasone propionate on human nasal mucosal surface and in human nasal tissue over a period of $24 \mathrm{~h}$ after intranasal application. Allergy, 56:532-5.

Bousquet J, Van Cauwenberge P, Khaltaev N. 2001. Allergic rhinitis and its impact on asthma. J Allergy Clin Immunol, 108(Suppl 5):S147-S334.

Bousquet J, Jacquot W, Vignola AM, et al. 2004. Allergic rhinitis: a disease remodeling the upper airways? J Allergy Clin Immunol, 113:43-9.

Canonica GW. 2006. Treating asthma as an inflammatory disease. Chest, 130:21S-8S.

Chapman KR, Patel P, D’Urzo AD, et al. 2005. Maintenance of asthma control by once-daily inhaled ciclesonide in adults with persistent asthma. Allergy, 60:330-7.

CKS. 2007. Allergic rhinitis. Clinical knowledge summaries [online. URL: http://www.cks.library.nhs.uk/allergic_rhinitis/view_whole_guidance.

Dent G. 2002. Ciclesonide (Byk Gulden). Curr Opin Investig Drugs, 3:78-83

Dietzel K, Engelstätter R, Keller A. 2001. Ciclesonide: an on-site-activated steroid, In: Hansel TT, Barnes PJ (eds). New drugs for asthma, allergy and COPD. Basel, Switzerland: Karger. p 91-3.

Drollmann A, Watz H, Nave R, et al. 2006. In vivo metabolism of ciclesonide in the human lung. Proc Am Thorac Soc, 3:A75.

Edsbäcker S, Brattsand R. 2002. Budesonide fatty-acid esterification: A novel mechanism prolonging binding to airway tissue. Review of available data. Ann Allergy Asthma Immunol, 88:609-16.

Gelfand EW. 2004. Inflammatory mediators in allergic rhinitis. $J$ Allergy Clin Immunol, 114:S135-S8.

Engelstätter R, Banerji D, Steinijans VW, et al. 2004. Low incidence of oropharyngeal adverse events in asthma patients treated with ciclesonide: results from a pooled analysis [abstract]. Am J Respir Crit Care Med, 169(Suppl). Abstract A92.

Esmailpour N, Hogger P, Rohdewald P. 2000. Binding of glucocorticoids to human nasal tissue in vitro. Int Arch Allergy Immunol, 122:151-4.

Gelfand EW. 2004. Inflammatory mediators in allergic rhinitis. J Allergy Clin Immunol, 114:S135-S8.

Georgitis J. 1999. The 1997 asthma management guidelines and therapeutic issues relating to the treatment of asthma. Chest, 115:210-7.

GINA Global Initiative for Asthma. 2005. National Institutes of Health, National Heart, Lung and Blood Institute. URL: www.ginasthma.com.

Gu Z, Zhao X, Nave R, et al. 2007. Comparative in vitro metabolism of 14C-ciclesonide in hepatocytes from the mouse, rat, rabbit, dog, and human. Am J Ther, 14:280-90.

Janciauskiene S, Orbjörn C, Sjölin P, et al. 2007. Corticosteroid lipophilicity is a more important determinant of corticosteroid uptake into human hepatocytes than serum protein binding. Eur Respir J, 30(Suppl 51):351S. 
Kim K, Weiswasser M, Nave R, et al. 2007. Safety of once-daily ciclesonide nasal spray in children with perennial allergic rhinitis. Pediatr Asthma Allergy Immunol, 20:229-42.

Langdon CG, Adler M, Mehra S, et al. 2005. Once-daily ciclesonide 80 or 320 microg for 12 weeks is safe and effective in patients with persistent asthma. Respir Med, 99:1275-85.

Lexmuller K, Gullstrand H, Axelsson BO, et al. 2007. Differences in endogenous esterification and retention in the rat trachea between budesonide and ciclesonide active metabolite. Drug Metab Dispos, 35:1788-96.

McCracken NW, Blain PG, and Williams FM, 1993. Human xenobiotic metabolising esterases in liver and blood. Biochem Pharmacol, 46:1125-9.

Mutch E, Nave R, McCracken N, et al. 2007. The role of esterases in the metabolism of ciclesonide to desisobutyryl-ciclesonide in human tissue. Biochem Pharmacol, 73:1657-64.

Nave R, Hummel RP, Wohlsen A, et al. 2004. The active metabolite of ciclesonide, des-isobutyryl ciclesonide, forms highly lipophilic fatty acid conjugates in precision-cut rat lung slices. Am J Respir Crit Care Med, 169(Suppl 7):A91.

Nave R, Zech K, Bethke TD. 2005a. Lower oropharyngeal deposition of inhaled ciclesonide via hydrofluoroalkane metered-dose inhaler compared with budesonide via chlorofluorocarbon metered-dose inhaler in healthy subjects. Eur J Clin Pharmacol, 61:203-8.

Nave R, Meyer W, Fuhst R, et al. 2005b. Formation of fatty acid conjugates of ciclesonide active metabolite in the rat lung after 4-week inhalation of ciclesonide. Pulm Pharmacol Ther, 18:390-6.

Nave R, Fisher R, Zech K. 2006a. In vitro metabolism of ciclesonide in human lung and liver precision-cut tissue slices. Biopharm Drug Dispos, 27:197-207.

Nave R., Wingertzahn MA, Brookman S, et al. 2006b. Safety, tolerability, and exposure of ciclesonide nasal spray in healthy and asymptomatic subjects with seasonal allergic rhinitis. J Clin Pharmacol, 46:461-7.

Nave R, Fisher R, McCracken N. 2007. In vitro metabolism of beclomethasone dipropionate, budesonide, ciclesonide, and fluticasone propionate in human lung precision-cut tissue slices. Respir Res, 8:65.

[NIH] National Institutes of Health: National Heart Lung and Blood Institute. 1997. National Asthma Education and Prevention Program Expert Panel Report 2. Clinical practice guidelines: Guidelines for the diagnosis and management of asthma. Bethesda, Maryland: National Institutes of Health/National Heart, Lung, and Blood Institute: NIH publication no. 97-4051.

Nonaka T, Sugiyama H, Sato H, et al. 2008. Prolonged anti-inflammatory activity of ciclesonide in human bronchial epithelial cells. SUBMITTED MANUSCRIPT.
Nonaka T, Nave R, McCracken N, et al. 2007. Ciclesonide Uptake and Metabolism in Human Alveolar Type II Epithelial Cells (A549). BMC Pharmacol, 7:12.

Peet CF, Enos T, Nave R, et al. 2005. Identification of enzymes involved in phase I metabolism of ciclesonide by human liver microsomes. Eur J Drug Metab Pharmacokinet, 30:275-86.

Petersen H, Kullberg A, Edsbacker S, et al. 2001. Nasal retention of budesonide and fluticasone in man: formation of airway mucosal budesonide-esters in vivo. Br J Clin Pharmacol, 51:159-63.

Postma DS, Sevette C, Martinat Y, et al. 2001. Treatment of asthma by the inhaled corticosteroid ciclesonide given either in the morning or evening. Eur Respir J, 17:1083-8.

Ratner PH, Wingertzahn MA, van Bavel JH, et al. 2006. Efficacy and safety of ciclesonide nasal spray for the treatment of seasonal allergic rhinitis. J Allergy Clin Immunol, 118:1142-8.

Richter K, Kanniess F, Biberger C, et al. 2005. Comparison of the oropharyngeal deposition of inhaled ciclesonide and fluticasone propionate in patients with asthma. J Clin Pharmacol, 45:146-52.

Rohatagi S, Luo Y, Shen L, et al. 2005. Protein binding and its potential for eliciting minimal systemic side effects with a novel inhaled corticosteroid, ciclesonide. Am J Ther, 12:201-9.

Ryrfeldt A, Andersson P, Edsbacker S, et al. 1982. Pharmacokinetics and metabolism of budesonide, a selective glucocorticoid. Eur $J$ Respir Dis Suppl, 122:86-95.

Sato H, Nave R, Nonaka T, et al. 2006. In vitro activation of the corticosteroid ciclesonide in animal nasal mucosal homogenates. Biopharm Drug Dispos, 28:59-64.

Sato H, Nave R, Nonaka T, et al. 2007a. In vitro metabolism of ciclesonide in human nasal epithelial cells. Biopharm. Drug Dispos, 28:43-50.

Sato H, Nave R, Nonaka T, et al. 2007b. Uptake and metabolism of ciclesonide and retention of desisobutyryl-ciclesonide for up to 24 hours in rabbit nasal mucosa. BMC Pharmacol, 7:7.

Skoner DP. 2001. Allergic rhinitis: definition, epidemiology, pathophysiology, detection, and diagnosis. J Allergy Clin Immunol, 108(Suppl): S2-S8.

Storms WW. 2004. Pharmacologic approaches to daytime and nighttime symptoms of allergic rhinitis. J Allergy Clin Immunol, 114: S146-S53.

Tunek A, Sjödin K, Hallström G. 1997. Reversible formation of fatty acid esters of budesonide, an anti-asthma glucocorticoid, in human lung and liver microsomes. Drug Metab Dispos, 25:1311-17.

Van den Brink KIM, van den Brekel IJS, Boorsma M, et al. 2005. Evidence of in vivo esterification of budesonide in humans. Eur Respir J Suppl, 26(Suppl 49):370s 